\title{
Editorial
}

\section{FACTOR DE IMPACTO Y NUEVAS HERRAMIENTAS DE USO DE CIRUGÍA PLÁSTICA IBEROLATINOAMERICANA}

El español es en la actualidad la tercera lengua en número de hablantes (5\% de la población mundial); es a su vez el idioma con mayor crecimiento ( $8 \%$ en los últimos 8 años); la segunda lengua mundial de intercambio económico y la tercera más hablada en Internet. Junto a ello, el otro idioma en el que publica Cirugía Plástica Iberolatinoamericana, el portugués, es la octava lengua mundial en número de hablantes y la tercera más usada entre los occidentales. Entre ambos idiomas reunimos a una comunidad de más de 600 millones de personas en el mundo que sueñan, piensan y se expresan en nuestras mismas lenguas.

Además de este incremento lingüístico, España y Brasil se encuentran entre los 5 países del mundo que más han crecido en producción científica en los últimos años.

Quiere esto decir que estamos en el camino de no tener que depender siempre de los baremos anglosajones que califican la importancia de una publicación científica o de su "mercado" editorial en base a la supuesta exclusividad de las revistas incluidas en la base de datos del SCI (Sciencie Citation Index) para poder desarrollar y dar a conocer nuestra actividad científica, ni por tanto referenciar como exclusivo el conocido Factor de Impacto de su propietario, el Thomsom Institute (ISI), único existente hasta no hace mucho tiempo (pero no por ello mejor, sino único, tal y como expresó con sus propias palabras su creador Eugene Garfield).

Con esa idea, hace ya tiempo que el grupo editorial Elsevier B.V. creó el portal Scopus ${ }^{\circledR}$ que agrupa casi 18.000 títulos de publicaciones científicas y elabora su propio índice de citaciones. Se trata de una base que funciona mediante suscripción, pero sobre la cual, el grupo SCImago®, en España, ha desarrollado un portal de acceso gratuito llamado SCImago Journal and Country Rank, activo desde 2006, a través del cual podemos tener libre acceso a todos los indicadores de visibilidad y difusión de las revistas científicas incluidas en la base Scopus.

No se trata de competir, sino de crear una vía paralela que sirva de referente internacional en la que, otras publicaciones no incluidas en el SCI básicamente por su idioma y difusión, puedan también ser acreditadas y medidas; en nuestro caso, ser referentes de la publicación sobre Cirugía Plástica, Estética y Reparadora hecha en español por una comunidad científica creciente (sin olvidar por supuesto el portugués).

Nuestra Revista, dado el crecimiento en calidad y visibilidad alcanzado en los últimos años, y con el impulso de su edición electrónica a través de la plataforma SciELO iniciada en
2006, está incluida tanto en la base Scopus como en SCImago, y por tanto disponemos de nuestro propio factor de impacto o SJR (Scientific Journal Rank en nomenclatura SCImago) dentro de esta base.

El SJR para Cirugía Plástica Iberolatinoamericana en 2008 nos da un valor de 0,03. Parafraseando a Winston Churchill cuando decía que "las estadísticas son como un bikini, muestran datos interesantes pero esconden lo realmente importante", vamos a dedicar esta nota editorial a analizar este frío dato numérico para saber qué significa realmente esa cifra para nuestra publicación.

El total de las publicaciones incluidas en el portal SCImago es de 17.124 revistas internacionales agrupadas en 28 áreas de interés de las cuales una es el área médica que incluye 49 especialidades. La primera revista en el SJR general es, por supuesto, una revista americana de Inmunología con valor 16,024. En el listado del SJR del área de interés Medicina, encontramos en primer lugar una revista americana sobre cáncer con valor 11,331; es curioso observar en ese listado que desde ese número, se desciende rápidamente hasta el valor 3,63 que aparece para la revista situada en el quinto puesto. Ya dentro del área puramente quirúrgica, en la que se encuentra nuestra revista, el primer lugar de la lista lo ocupa una revista americana sobre investigación ósea con valor 0,818 ; nuevamente observamos como desde ahí se produce un rápido descenso, de manera que la revista que ocupa el cuarto puesto tiene ya un valor de la mitad, 0,45. Empezamos a extraer apuntes de estas lecturas: son muy pocas las revistas que tienen valores SJR muy altos y generalmente están dedicadas a temas que interesan a comunidades científicas muy amplias, lo que incrementa notablemente su difusión, accesibilidad y visibilidad.

Si seguimos buscando dentro del listado quirúrgico general compuesto por 220 publicaciones, la más afamada revista científica en nuestro medio, Plast. \& Rec. Surg., aparece en el puesto 34 con un valor SJR de 0,154 . Otras revistas del medio que nos suelen servir de referente son Annals Plast. Surg, con 0,11, Clin. Plast. Surg. con 0,08, Scand. J. Plast. Surg. con 0,05 y Aesth. Surg. J. con 0,04. Nuestra revista aparece en el puesto 169 con valor 0,03 ; vemos por tanto que su valor SJR no está demasiado alejado del de otras relativas a nuestra especialidad.

Si seguimos analizando, de ese total de 220 solo 4 revistas quirúrgicas son españolas y de ellas la nuestra es la segunda en impacto tras la revista Cirugía Española que tiene un valor de 
0,047 y ocupa el puesto 126 entre las revistas quirúrgicas. Por detrás de nosotros estarían la Revista Española de Cirugía Ortopédica y Traumatología con 0,028 y, siendo además la más cercana por materia a nuestro ámbito de difusión, aparece en cuarto lugar la Revista de Cirugía Oral y Máxilofacial, con valor 0,027.

Si comparamos Cirugía Plástica Iberolatinoamericana, con una edición electrónica de 16 ejemplares desde 2006 y la revista española de Cirugía Oral y Máxilofacial, con 36 ejemplares en su edición electrónica desde 2003, podremos entender mejor nuestra rápida progresión en un corto espacio de tiempo.

Es importante también dar a conocer que el SJR de SCImago tiene una particularidad muy interesante; considera no solo el número de citas que recibe un artículo, sino también la importancia o influencia de las publicaciones que hacen esas citas; es decir, valora el impacto científico no solo en cuanto a la cantidad de citas recibidas, sino combinando cantidad y calidad de las publicaciones que hacen esas citas, cosa que no hace el tradicional Factor del Impacto del SCI que mide solo datos numéricos de cantidad, muchas veces contaminados por las variaciones en accesibilidad, visibilidad y difusión de que dispone cada publicación en función de sus medios económicos, además de por la tradicional tendencia a la auto-referencia endogámica promocionada por algunas prestigiosas publicaciones internacionales de nuestro sector.

La idea es que con todos estos datos, aprendamos a resaltar la existencia de nuevas herramientas internacionales de baremación de revistas científicas para que toda esta información, disponible en acceso libre a todos los que quieran consultarla, sea tenida en cuenta a la hora de puntuar como es debido las publicaciones que nuestros autores hacen en nuestro propio idioma y no solo en las revistas publicadas en inglés, más difundidas, eso sí, pero no por ello con el signo exclusivo de calidad.

El futuro de la publicación científica en nuestro idioma depende por tanto de todos nosotros: FILACP, Sociedades Nacionales, Servicios Docentes con formación acreditada, Universidades, Colegios profesionales y por supuesto, de todos y cada uno de los especialistas en Cirugía Plástica, Estética y Reparadora a los que nos une una misma y pujante comunidad lingüística.

Debemos centrar nuestros esfuerzos en no ser consumidores pasivos de Ciencia, sino en comunicar por escrito lo que sabemos y lo que hacemos a diario en nuestra especialidad y ver en
Cirugía Plástica Iberolatinoamericana la vía natural para esa comunicación. Crear terminología propia evitando traducciones simplistas y anglicismos innecesarios en nuestros artículos. Referenciar en nuestros escritos o presentaciones lo que publicamos o leemos en nuestra propia Revista que es mucho e importante y exigir que se acredite y bareme adecuadamente el bagaje de publicaciones hechas en español en nuestros CV.

Destacar también que Cirugía Plástica Iberolatinoamericana ha sido incluida en el rastreador Google Académico, dedicado exclusivamente a la búsqueda de publicaciones científicas en Internet. Esto nos permite acceder directamente, en una búsqueda bibliográfica por materias o por autores, a artículos publicados en nuestra Revista, tanto en su formato electrónico desde 2006, como en los años anteriores en su edición impresa. Gracias a estar integrados en la red SciELO y a las etiquetas adjuntas que cada artículo publicado lleva incluidas en dicha red, nos proporciona además en cada trabajo datos sobre las veces que ese artículo ha sido citado en otra publicación, quién lo ha citado, en qué revista y el enlace a otros artículos relacionados, con lo que también podemos fácilmente encontrar de esta manera citas a artículos de nuestra Revista anteriores a 2006 y por tanto localizarlos de forma sencilla en la edición impresa que todos coleccionamos.

Invitamos a todos los lectores a explorar las nuevas herramientas que a lo largo de 2009 se han ido incorporando a nuestra edición electrónica a través de SciELO: registro propio de ISSN 0376-7892, enlace a SJR y Google Académico, citación directa, envío de artículos a través de internet e incluso un traductor automático que permite a lectores de otros países interesados en nuestros artículos a través de su resumen en inglés, poder leerlos directamente también en este idioma en su versión completa. Desde marzo de 2010 cada artículo dispone además de su propio DOI o Número de Identificación de Artículos Electrónicos, marcador universal y a perpetuidad de todos y cada uno de los artículos publicados que asegura su localización y seguimiento indefinido aunque en el futuro las bases de datos que ahora los contienen desaparecieran.

Finalizar diciendo que también la red MEDES de revistas médicas en español de la Fundación Lilly ha incluido un enlace a la edición electrónica de Cirugía Plástica Iberolatinoamericana desde finales de 2009.

Todos los datos expuestos pueden encontrarse entrando en:

$$
\begin{aligned}
& \text { http://scielo.isciii.es/scielo.php?script=sci_serial\&pid=0376-7892\&lng=es\&nrm=iso } \\
& \text { http://www.scimagojr.com/journalsearch.php?q=03767892\&tip=iss\&exact=yes } \\
& \text { http://scholar.google.es/ } \\
& \text { http://www.lilly.es/Nitro/foundation/templates/lines.jsp?page=30640 }
\end{aligned}
$$

